\title{
UNDER PRESSURE - HOW TIME PRESSURE AFFECTS AUTOMATION-AIDED DECISION-MAKING IN A LUGGAGE SCREENING TASK
}

\author{
Tobias Rieger, Technische Universität Berlin \\ Dietrich Manzey, Technische Universität Berlin
}

Automation and specifically computer-based automated assistance systems are present in virtually every field today. One special case of automation are decision support systems (DSSs) which are thought out to aid a human execute a certain task. However, even with highly reliable systems, the resulting performance consequences of this automation support is less than optimal (Bartlett \& McCarley, 2017). One reason is that trust (and accordingly, compliance and reliance) are often not properly calibrated causing decision aids to be misused or disused (Parasuraman \& Riley, 1997). The degree of time pressure which operators have in making their decisions while interacting with an automated DSS might make a difference in this respect. Previous research (Rice \& Keller, 2009) has found that time pressure tends to increase dependence on the DSS, possibly even improving overall performance if the DSS is highly reliable. The goal of the present experiment was therefore to examine the influence of time pressure on the use of an automated DSS and related human performance consequences, dependent on the reliability of the aid. To this end, we used a luggage screening task which had to be performed either manually, with support of a low reliability DSS (75\%), or with support of a high reliability DSS (95\%). In essence, we used false-alarm prone systems because this resembles real-world alarm systems in safety critical environments where one tries to detect any signal. A total of 60 participants (all novices with respect to the task) took part in the experiment and were randomly assigned to one of the three DSS conditions (high reliability, low reliability, manual). Participants worked on a luggage-screening task under both $\mathrm{high} /$ low time pressure (indicated by a countdown) which was varied blockwise within-subjects. We hypothesized that participants in the high reliability DSS condition had better performance than those in the low reliability condition and that this difference should become even more pronounced with time pressure. The manual condition mainly served as a control condition. Main dependent variables were the percentage of correct responses, as well as compliance and reliance rates in interaction with the DSS. Contrasting to earlier findings, we did not find an increased dependence on the automation under high time pressure and, thus, also no performance increases. Our key findings were that (a) time pressure led to worse overall performance, (b) this negative effect of time pressure was not attenuated (or even lead to positive effects) when a highly reliable automation was available, and (c) that performance increased with a highly reliable automation but was still worse than the automation alone. Moreover, participants' compliance did not differ between low and high time pressure, but reliance decreased under high time pressure. To reconcile the present findings, it seems that time pressure is rightfully often considered as a to- be-avoided workload factor. Moreover, the present findings reinforce earlier concerns about joint human-automation performance with a highly reliable system.

References

Bartlett, M. L., \& McCarley, J. S. (2017). Benchmarking aided decision making in a signal detection task. Human Factors,59(6), 881-900. doi: 10.1177/0018720817700258

Parasuraman, R., \& Riley, V. (1997). Humans and automation: Use, misuse, disuse, abuse. Human Factors, 39(2), 230-253. doi: 10.1518/001872097778543886

Rice, S., \& Keller, D. (2009). Automation reliance under time pressure. Cognitive Technology, 14(1), 36-44. 